Original Research Paper

\title{
An Economic System for Screening of Diabetic Retinopathy Using Fundus Images
}

\author{
${ }^{1}$ G.S. Annie Grace Vimala and ${ }^{2}$ S. Kaja Mohideen \\ ${ }^{I} E C E$, St. Joseph's Institute of Technology, Chennai, India \\ ${ }^{2} E C E$, B.S. Abdur Rahman University, Chennai, India
}

\author{
Article history \\ Received: 31-08-2014 \\ Revised: $13-09-2014$ \\ Accepted: 26-09-2014 \\ Corresponding Author: \\ G.S. Annie Grace Vimala \\ ECE, St. Joseph's Institute of \\ Technology, Chennai, India \\ Email: gsannie77@gmail.com
}

\begin{abstract}
Delayed length of time of the diabetes may influence the tiny blood vessels of the retina bringing about Diabetic Retinopathy (DR). It is one of the major root causes of the blindness across the globe. Routine eye screening of patients with diabetes serves to discover DR at the early stage. The conventional visual assessment protocol is time consuming and laborious. Hence, the computer aided system for DR detection can reduce the burden of the ophthalmologist and improve the accuracy. In this work, we have proposed an automatic method for screening exudates based on image processing methods which utilized color component, morphology and intensity in retinal digital fundus images. The retinal fundus image of the affected eye was recorded with a fundus camera (Visupac, CARLZEISS FF 450 plus) for 60 south Indian women. For each image the ground truth result was collected from two glaucoma experts with more than 10 years of experience. Then the quantitative evaluation of the proposed algorithm has been carried out using these ground truth results. We achieved the highest segmentation accuracy of $93 \%$, F-score of $88.2 \%$, precession and recall of 93.1 and $89.3 \%$ respectively when the ground truth results were considered as standard. The extracted morphological parameters exhibited significant differences between normal and DR groups at $\mathrm{p}<0.001$, whereas, the age and BMI values depicted no such significance. In conclusion, our findings suggest that the proposed system would be a useful for mass screening of DR.
\end{abstract}

Keywords: Fundus Image, Diabetic Retinopathy, Image Processing, Mathematical Morphology, Statistical Analysis

\section{Introduction}

The rapid growth of diabetes is one of the greatest challenges of the existing health services department. The percentage of human subjects affected with the sickness keeps on rising at a quick rate. Three hundred and forty five million human subjects are suffering from diabetes across the globe; among them 41 million were Indians, reported by International Diabetic Federation (IDF, 2012). About $40 \%$ of human subjects with diabetes have at least of mild signs of Diabetic Retinopathy (DR) (Nayak et al., 2008). The DR is damage to the retina initiated by problems of diabetes, which can finally lead to loss of vision or blindness. It could be a mute illness; thus, it should merely be recognized by the patient once the progressions inside the retina have advanced to the level wherever treatment is difficult or much complicated (Kinyoun et al., 1989; Bresnick et al., 2001). Macular edema and proliferative retinopathy are two major mechanisms by which the vision is lost due to DR. Macular edema is the increase of fluid called exudates in macula and which is the main cause of vision loss and its prevalence is set to continue rising. Exudates are proteins and lipids that deposits and leaks from the damaged blood vessels within the retinal region; they are whitish-yellow lesions with relatively distinct margins. Detection of exudates by ophthalmologists is a difficult process as they have to spend a great deal of time in manual analysis (Sopharak et al., 2009). In addition, manual 
detection involves utilization of chemical dilation material which takes more time and has harmful side effects on the patients. So as to minimize the side effects and cost of such screenings, a digital image acquiring technology ought to be utilized (Lin et al., 2002; Williams et al., 2004). This technology allows the scientists to utilize the 'state-of-the art' image processing techniques which automate the detection of abnormalities. Hence, the aim of the present study was to extract various morphological features from retinal digital fundus images in the assessment of DR.

\section{Materials and Methods}

The detailed flow of the proposed study is outlined in the Fig. 1. This contains the information about study design, data collection and descriptions about steps involved in image processing implementation such as pre-processing, segmentation and morphological features extraction as well as statistical validation of the results with ground truth results.

\section{Data Collection}

A medical screening camp related to eye disease was organized during the end of year 2012 in an eye care hospital, Chennai, India. Sixty south Indian women (mean \pm SD age $=60.4 \pm 10.2$ years, range $=50-85$ years $)$ were participated in the camp and included for the present study. Women with vision loss and those who are under medication were excluded. A detailed questionnaire contains information about demographic, anthropometric as well as clinical history was filled and signed informed voluntary consent form by each participant participated in the camp.

For each woman, a digital image of the affected eye was acquired using a digital fundus camera (FF 450 plus, Carl Zeiss Meditec, Inc, USA) in combination with Visupac image management software system. The acquired digital fundus images were of size $640 \times 480$ and stored in the local hard drive of a computer system for further analysis. Then the stored images were visually analyzed by an experienced ophthalmologist and grouped them into (i) normal group and (ii) diabetic retinopathy group.

\section{Image Processing}

The pre-processing consists of three stages: (i) Color normalization, (ii) edge enhancement and (iii) color space conversion. Digital fundus image of each woman was pre-processed using the procedures detailed in (Sapthagirivasan and Anburajan, 2013) and our previous study (Vimala and Kajamohideen, 2014). The next step was binarizing the pre-processed fundus images. The binarisation process was done by setting an empirical threshold value of 0.78 . The binary image contains many objects such as blood vessels, optic disc, exudates and some unwanted noises in the retinal region. Based on removing the following contents from the binary image exudates contents were retained (Vimala and Kajamohideen, 2014):

- The blood vessels contain not much information about exudates

- Optic disc which is the entry region of blood vessels and optic nerve to the retina

- Borders of the retinal region

Finally, the following morphological features detailed by Sopharak et al. (2009; Vimala and Kajamohideen 2014) were extracted from the binary image which contains only small objects information:

Area

It was calculated based on total number of white pixels in the binary image.

\section{Number of Objects}

It was the count of total number of pieces in the binary image.

\section{Eccentricity}

It was estimated based on major and minor axis lengths of segmented pieces respectively.

\section{Extent}

It is the ratio between area and bounding box of the segmented piece.

\section{Orientation}

It is defined as the angle between major axis of the segmented piece that has the same second moments around the region and the horizontal axis.

\section{Convex Area of the Polygon}

It is calculated based on the area of the polygon around the segmented piece.

\section{Solidity}

It could be defined as the ratio of the solid or filled area and convex hull area of the segmented piece.

\section{Statistical Analysis}

The demographic features and extracted morphological features were analyzed using SPSS software package version 17.0 (SPSS Inc., Chicago, USA). The measured values in each subgroup were compared using a student's t-test. The data represented were mean $\pm \mathrm{SD}$. 


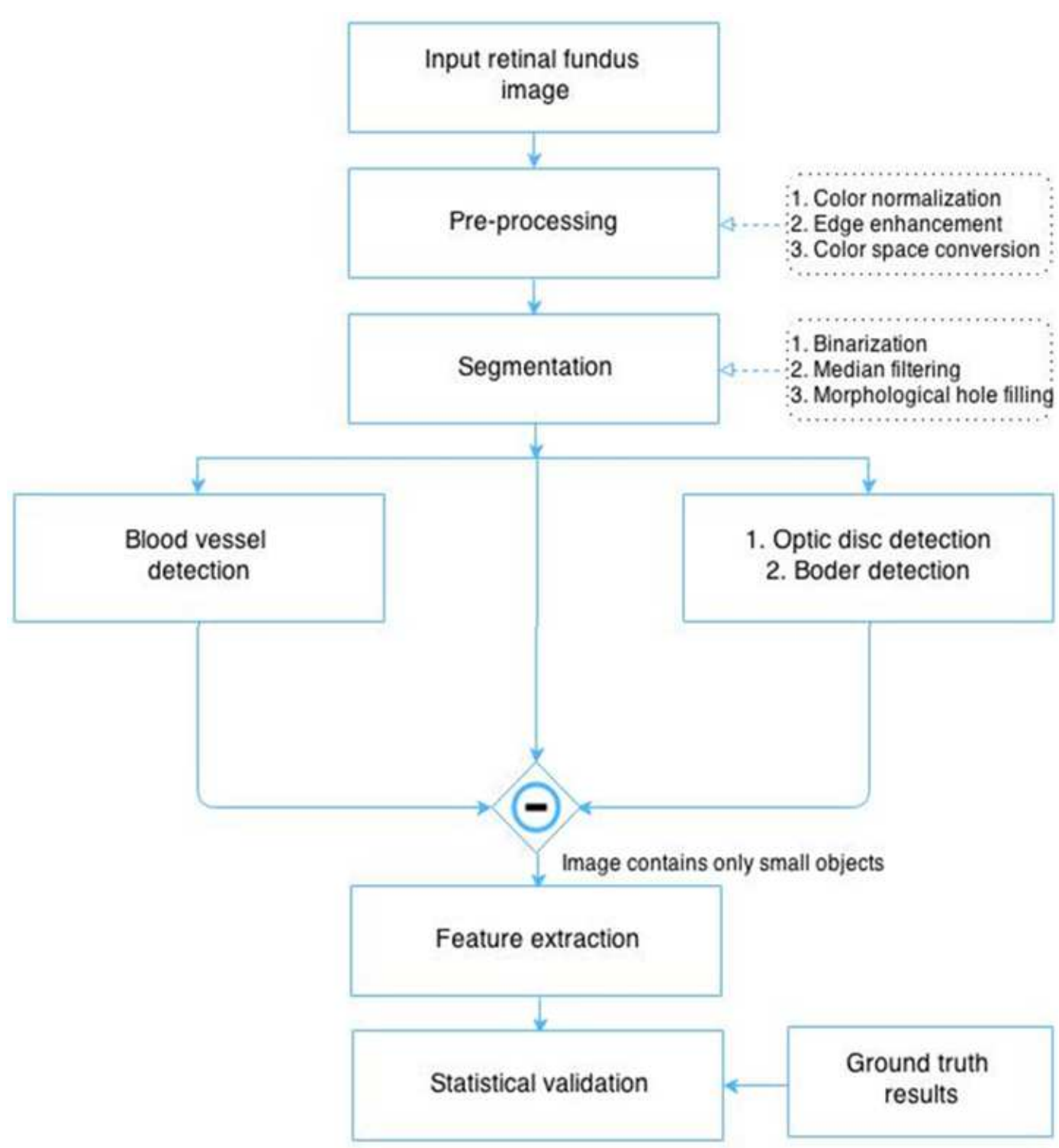

Fig. 1. Flow diagram of the proposed diabetic retinopathy detection system

\section{Results}

All retinal fundus images are acquired using a standard protocol. For each image the ground truth result was collected from two glaucoma experts with more than ten years of experience. Then the quantitative evaluation of the proposed algorithm has been carried out using these ground truth results. Out of the 60 studied women, $43 \%(26 / 60$, mean $\pm \mathrm{SD}$ age $=53.2 \pm 8.4$ years $)$ were belongs to normal group and $57 \%(34 / 60$, mean \pm SD age $=57.2 \pm 11.4$ years) were belongs to DR group based on the ground truth classification.

The retinal fundus images of the normal and DR samples are displayed in Fig. 2. The feature extraction technique detailed in the methodology section has been applied to each fundus image and results obtained are depicted in the Table 1 . The Table 1 also contains the details about the features extracted using the hand drawn ground truth results along with the demographic as well as anthropometric parameters. The age and Body Mass Index (BMI) values were not showing any significant variation between normal and DR group. Whereas, the morphological features extracted from digital fundus images using ground truth method as well as automatic image analysis algorithm exhibited significant $(p<0.001)$ difference between the groups i.e. the mean intensity value displayed significance increment in DR group when compared to normal group. The results of various 
steps involved in the segmentation of exudates region is shown in Fig. 3.

We have compared the performance of our proposed exudates extraction technique with the ground truth method. To assess the area overlap between the computed regions and the ground truth results, the pixelwise precision and recall values are computed. These are defined as follows Equation 1 and 2:

Precession $=T P /(T P+F P)$

Recall $=T P /(T P+F N)$ where, $T P, F P$ and $F N$ are number of true positive, false positive and false negative pixels respectively. Finally, we have computed traditional $F$-score and segmentation accuracy using the following Equation 3 and 4:

$$
F=\frac{\text { Precision } \times \text { Recall }}{\text { Precession }+ \text { Recall }}
$$

$$
\text { Accuracy }=\left[1-\frac{\text { Number of misclassified pixels }}{\text { Total pixels }}\right] \times 100
$$

The result of the proposed exudates detection process has depicted in Table 2. The system achieved 93\% segmentation accuracy with the ground truth results.

\begin{tabular}{|c|c|c|c|}
\hline Variables/groups & $\begin{array}{l}\text { Normal group }(\mathrm{n}=26) \\
\text { Mean } \pm \text { SD }\end{array}$ & $\begin{array}{l}\text { DR group }(\mathrm{n}=34) \\
\text { Mean } \pm \mathrm{SD}\end{array}$ & $P$ value \\
\hline \multicolumn{4}{|c|}{ Demographic and anthropometric parameter } \\
\hline Age (years) & $53.2 \pm 8.4$ & $57.2 \pm 11.4$ & 0.152 \\
\hline BMI $\left(\mathrm{kg} / \mathrm{m}^{2}\right)$ & $28.3 \pm 5.3$ & $29.2 \pm 7.4$ & 0.184 \\
\hline \multicolumn{4}{|c|}{ Retinal image parameters extracted by 'ground truth' } \\
\hline Mean intensity (gray) ${ }^{\neq}$ & $0.52 \pm 0.12$ & $0.82 \pm 0.06$ & $<0.000$ \\
\hline Mean area $(\%)$ & $50.5 \pm 14.2$ & $12.7 \pm 8.9$ & $<0.000$ \\
\hline Number of objects & $40.7 \pm 11.4$ & $17.3 \pm 4.5$ & $<0.000$ \\
\hline Solidity & $0.18 \pm 0.06$ & $0.71 \pm 0.01$ & $<0.000$ \\
\hline \multicolumn{4}{|c|}{ Retinal image parameters extracted by automated system } \\
\hline Mean intensity (gray) $^{\neq}$ & $0.59 \pm 0.18$ & $0.79 \pm 0.12$ & $<0.000$ \\
\hline Area & $46.6 \pm 11.4$ & $14.2 \pm 9.3$ & $<0.000$ \\
\hline Number of objects & $42.3 \pm 12.2$ & $15.6 \pm 4.9$ & $<0.000$ \\
\hline Solidity & $0.21 \pm 0.11$ & $0.76 \pm 0.03$ & $<0.000$ \\
\hline
\end{tabular}

Table 1. Range of the morphological features extracted from the retinal images

${ }^{\mp}$ Normalized the intensity values between 0 and 1; DR-Diabetic Retinopathy

Table 2. Result of exudates detection technique when comparing with ground truth method

\begin{tabular}{llll}
\hline Precession & Recall & F-score & Segmentation accuracy (\%) \\
\hline 0.931 & 0.893 & 0.882 & 92.62 \\
\hline
\end{tabular}

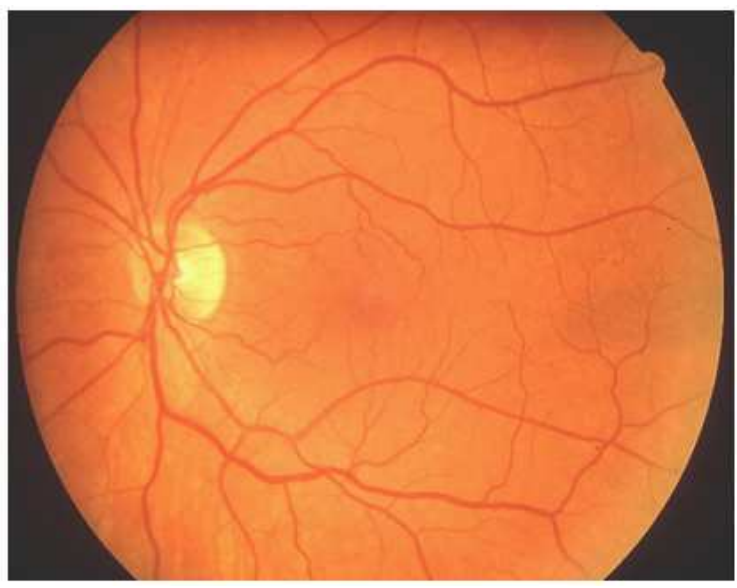

(a)

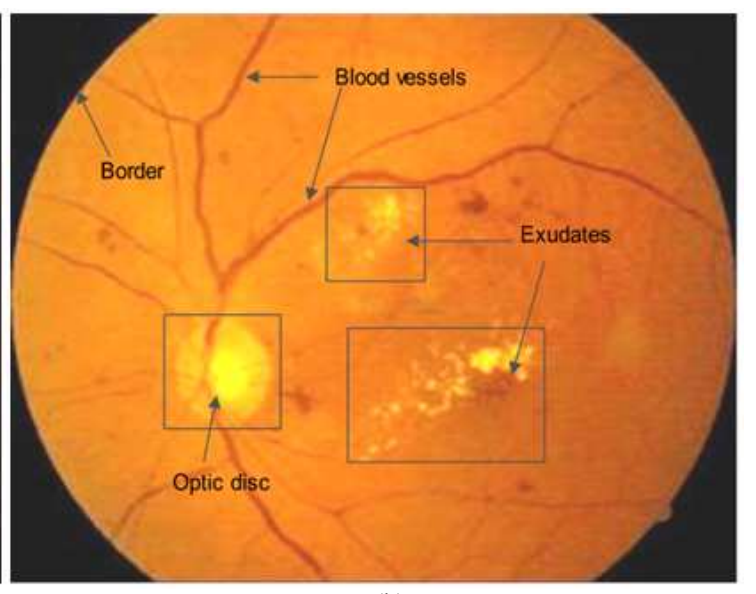

(b)

Fig. 2. Sample retinal fundus images of (a) Normal and (b) Diabetic retinopathy subjects 

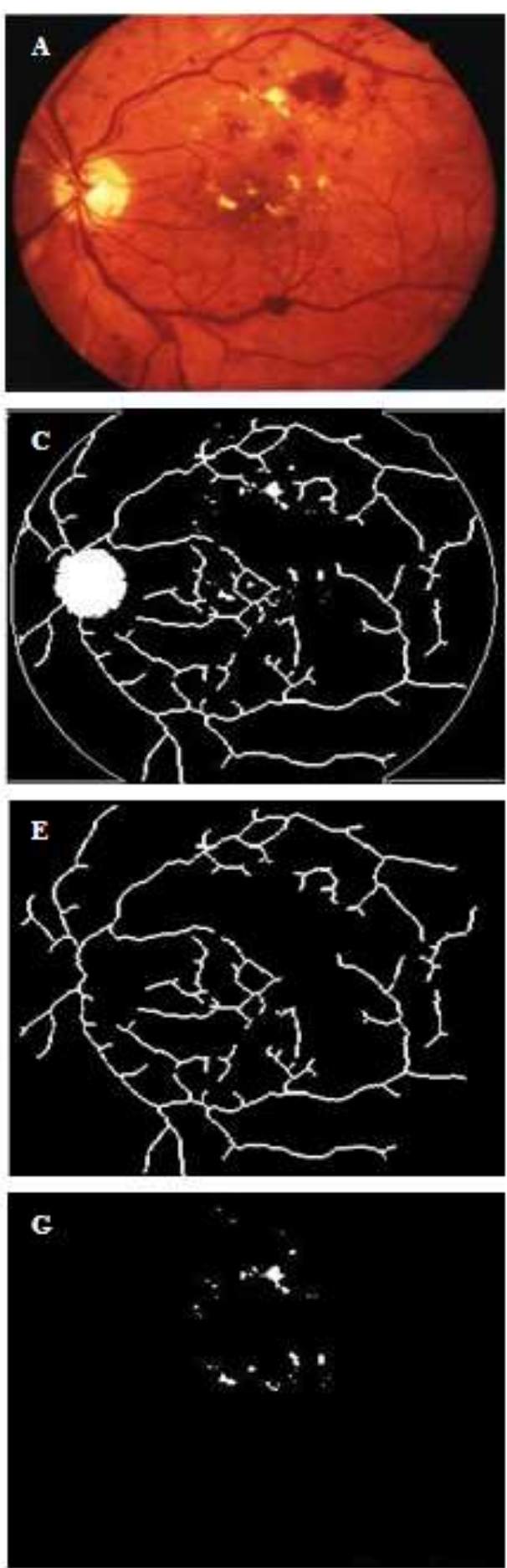
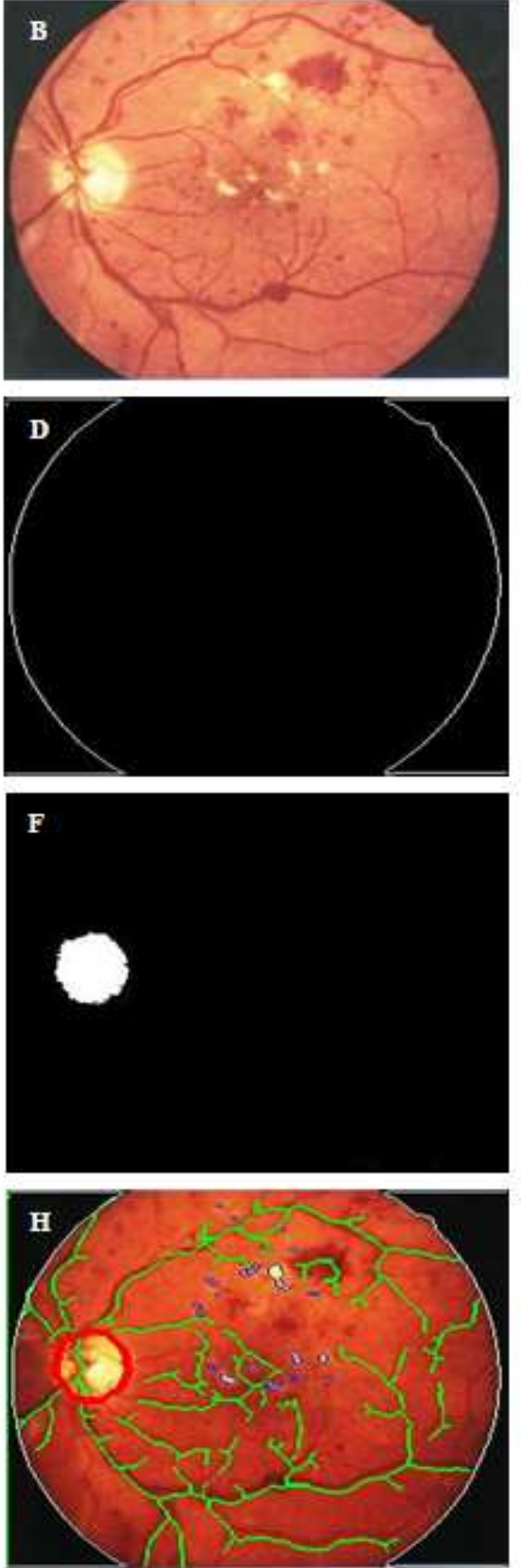

Fig. 3. Steps involved in the segmentation process. (a) input fundus image, (b) normalized result of (a), (c) segmented image, (d) border of (a), (e) extracted vessel image, (f) segmented optic disc, (g) exudates region $(c-(d+e+f)$ ), (h) super imposed image

\section{Discussion}

The World Health Organization predicts that in the next 25 years, the amount of individuals with diabetes across the globe will become double (Danaei et al.,
2011). About $50 \%$ of the individuals who are suffering from diabetes across the globe do not undergo any type of eye examination (Abramoff et al., 2008). This is due to lack of awareness form one side and the other side is problems in the conventional diagnosis procedures such 
as computational time as well as cost. So, there is an immediate need of developing mass screening tool which would work on bulk databases with affordable cost.

The proposed computer aided detection system is advantageous on account of its low cost, utilization of widely available fundus images and simple protocol. The fundamental behind the proposed system was the extraction of morphological parameters of exudate regions by subtracting blood vessels, optical disc and outer border of the retinal region. The extracted morphological parameters exudates region indicated the subtle changes in the normal and DR group. It can be clearly seen from Table 1 that all these morphological parameters show the significant difference value for normal as compared to the DR class $(p<0.001)$. For the normal images, the intensity values in the segmented region was observed as low (Table 1), whereas it was high for DR images due to the presence of hard exudates. The similar kind of variations was observed when the energy values extracted using wavelet based approach (Noronha et al., 2013). Similarly, the morphological parameters extracted from digital X-ray images for classification of osteoporosis form normal group also displayed significance at $\mathrm{p}<0.001$ (Sapthagirivasan et al., 2013). The age and BMI values were not shown any kind of significance between normal and DR group. Hann et al. (2009) explored the structural characteristics of the morphological features from digital fundus image and found the amount of pixels covering exudates could be used as a metric for the degree of the disease. Similarly, in the present study result in terms of accuracy (93\%) depicted in Table 2 was typical over all images with the number of exudates found agreeing well visually. The obtained result showed the potential for tracking the progress of DR. Similarly, the other parameters such as traditional F-score, precession and recall values were 88.2, 93.1 and $89.3 \%$ respectively.

Limitation of our study: The percentage of pixels covering exudates were used as a metric for the degree of the Disease (DR), hence this application requires further clinical validation with larger samples and specialists in the future.

\section{Conclusion}

Automatic methods for screening exudates for DR diagnosis has been developed based on image processing methods which utilized color component, morphology and intensity values in retinal digital fundus images. The extracted morphological parameters exhibited significant differences between normal and DR groups at $\mathrm{p}<0.001$, whereas, the age and BMI values depicted no such significance. The proposed system achieved 93\% segmentation accuracy of the exudates region form digital fundus images when ground truth (hand drawn) results were considered as standard. In addition, the traditional F-score, precession and recall values were 88.2, 93.1 and $89.3 \%$ respectively. However, these results need to be validated in future clinical trials.

\section{Author's Contributions}

All authors equally contributed in this work.

\section{Ethics}

This article is original and contains unpublished material. The corresponding author confirms that all of the other authors have read and approved the manuscript and no ethical issues involved.

\section{References}

Abramoff, M.D., M. Niemeijer, M.S.A. SuttorpSchulten, M.A. Viergever and S.R. Russell et al., 2008. Evaluation of a system for automatic detection of diabetic retinopathy from color fundus photographs in a large population of patients with diabetes. Diabetes Care, 31: 193-198.

DOI: $10.2337 / \mathrm{dc} 07-1312$

Bresnick, G.H., D.B Mukamel, J.C. Dickinson and D.R. Cole, 2000. A screening approach to the surveillance of patients with diabetes for the presence of vision threatening retinopathy, Ophthalmology, 107: 19-24.

Danaei, G., M.M. Finucane, Y. Lu, G.M. Singh and M.J. Cowan, et al., 2011. National, regional and global trends in fasting plasma glucose and diabetes prevalence since 1980: Systematic analysis of health examination surveys and epidemiological studies with 370 country-years and 2.7 million participants. Lancet, 378: 31-40. DOI: 10.1016/S0140-6736(11)60679-X

Hann, C.E., J.A. Revie, D. Hewett, J.G. Chase and G.M. Shaw, 2009. Screening for diabetic retinopathy using computer vision and physiological markers. J. Diabetes Sci. Technol., 3: 819-34. DOI: $10.1177 / 193229680900300431$

IDF, 2012. The International Diabetes Federation.

Kinyoun, J., F. Barton, M. Fisher, L. Hubbard and L. Aiello et al., 1989. Detection of diabetic macular edema. Ophthalmoscopy versus photography-early treatment diabetic retinopathy study report number 5. The ETDRS research group. Ophthalmology, 96: 746-750. PMID: 2740076 
Lin, D.Y., M.S. Blumenkranz, R.S. Brothers and D.M. Grosvenor, 2002. The sensitivity and specificity of single-field nonmydriatic monochromatic digital fundus photography with remote image interpretation for diabetic retinopathy screening: A comparison with ophthalmoscopy and standardized mydriatic color photography. Am. J. Ophthalmol., 134: 204-213. DOI: 10.1016/S0002-9394(02)01522-2

Nayak, J., P.S. Bhat, U.R. Acharya, C.M Lim and M. Kagathi, 2008. Automated identification of diabetic retinopathy stages using digital fundus images. J. Med. Syst., USA, 32: 107-115. PMID: 18461814

Noronha, K., U.R. Acharya, K.P. Nayak, S. Kamath and S.V. Bhandary, 2013. Decision support system for diabetic retinopathy using discrete wavelet transform. Proc. Inst. Mech. Eng. H., 227: 251-61. DOI: $10.1177 / 0954411912470240$

Sapthagirivasan, V. and M. Anburajan, 2013. Diagnosis of osteoporosis by extraction of trabecular features from hip radiographs using support vector machine: An investigation panorama with DXA. Comput. Biol. Med., 43: 1910-1919.

DOI: 10.1016/j.compbiomed.2013.09.00
Sapthagirivasan, V., M. Anburajan, V. Madhavan and T, Angu, 2013. The prominence of local contrast enhancement transformation approach in osteoporotic evaluation. WSEAS Trans. Biol. Biomed., 10: 22-34.

Sopharak, A., B. Uyyanonvara and S. Barman, 2009. Automatic exudate detection from non-dilated diabetic retinopathy retinal images using fuzzy Cmeans clustering. J. Sens., 9: 2148-2161. DOI: $10.3390 / \mathrm{s} 90302148$

Vimala, G.S.A.G. and S. Kajamohideen, 2014. Diagnosis of diabetic retinopathy by extracting blood vessels and exudates using retinal color fundus images. WSEAS Trans. Biol. Biomed., 11: 20-28.

Williams, G.A., I.U. Scott, J.A. Haller, A.M. Maguire and D. Marcus et al., 2004. Single-field fundus photography for diabetic retinopathy screening: A report by the American Academy of Ophthalmology. Ophthalmology, 111: 1055-1062. DOI: $10.1016 /$ j.ophtha.2004.02.004 